\title{
Chiral Perturbation Theory for Three-Flavour Lattice QCD with Isospin Splitting
}

\author{
S. Engelnkemper and G. Münster \\ Institut für Theoretische Physik, Universität Münster, Wilhelm-Klemm-Straße 9, 48149 Münster, Germany \\ Correspondence should be addressed to G. Münster; munsteg@uni-muenster.de
}

Received 1 March 2016; Accepted 3 May 2016

Academic Editor: Juan José Sanz-Cillero

Copyright (C) 2016 S. Engelnkemper and G. Münster. This is an open access article distributed under the Creative Commons Attribution License, which permits unrestricted use, distribution, and reproduction in any medium, provided the original work is properly cited. The publication of this article was funded by SCOAP ${ }^{3}$.

\begin{abstract}
An important tool for the analysis of results of numerical simulations of lattice QCD is chiral perturbation theory. In the Wilson chiral perturbation theory the effects of the finite lattice spacing $a$ are taken into account. In recent years the effects of isospin splitting on the masses of hadrons have been investigated in the Monte Carlo simulations. Correspondingly, in this paper we derive the expansions of the masses of the pseudoscalar mesons in chiral perturbation theory at next-to-leading order for twisted mass lattice QCD with three light quark flavours, taking the mass difference between the up- and downquarks into account. The results include terms up to orders $m_{q}^{2}$ in the quark masses, $\Delta m^{2}$ in the mass splitting between up- and downquarks, and $a^{2}$ in the lattice spacing, respectively.
\end{abstract}

\section{Introduction}

For investigations of QCD by means of numerical simulations on a lattice, chiral perturbation theory has become an indispensable tool. It allows describing the dependence of hadron masses and matrix elements on the quark masses in terms of expansions in powers of the quark masses, modified by logarithmic terms. On the one hand this offers the possibility to extrapolate the results of numerical simulations with larger quark masses into the physical regime with small up- and downquark masses. On the other hand, comparing the quark mass dependence observed in Monte Carlo calculations with the formulae from chiral perturbation theory yields the values of fundamental low-energy constants of QCD, the Gasser-Leutwyler coefficients. For reviews see $[1,2]$.

The discretisation of space-time on a lattice with lattice spacing $a$ implies a breaking of chiral symmetry and leads to discretisation effects in observable quantities. In the case of lattice QCD with the Wilson fermions the latter are generally of order $a$. A way to reduce lattice artifacts is to employ the formulation of twisted mass lattice QCD, which implements a chirally rotated mass term $[3,4]$. Setting the twist angle to the value $\pi / 2$ ("full twist") implies automatic $\mathcal{O}(a)$ Symanzik improvement $[5,6]$. The ETM collaboration has done simulations with two mass-degenerate quark flavours, representing the up- and downquarks, and with $2+1+1$ quark flavours, meaning a degenerate doublet of up- and downquarks and a nondegenerate doublet of charm and strange quarks, with full twist in both sectors [7-9].

Chiral perturbation theory for twisted mass lattice QCD has been developed in [10-13] for $N_{f}=2$ quark flavours. It has been extended to $N_{\mathrm{f}}=1+1$ nondegenerate quarks and $N_{\mathrm{f}}=$ $2+1$, including the strange quark, in [14], and to $N_{\mathrm{f}}=2+1+1$, with a degenerate doublet of up- and downquarks, in [15] and in [16] in the form of a charmless chiral Lagrangian. The resulting formulae for meson masses reveal the dependence on the lattice spacing $a$ in the form of additional terms proportional to powers of $a$. Chiral perturbation theory taking lattice effects for the Wilson fermions into account is called the Wilson chiral perturbation theory.

In recent years the isospin splitting produced by the small mass difference between up- and downquarks has come into the focus of numerical investigations of lattice QCD 
[17-19]. This splitting contributes to the mass splittings between charged and neutral hadrons. An additional source for these mass splittings is electromagnetic effects, which have been studied in the framework of lattice QCD + QED in [1821].

The purpose of this paper is to take the mass splitting between the light quark masses into account in the Wilson chiral perturbation theory for twisted mass lattice QCD with $N_{\mathrm{f}}=1+1+1$ quark flavours. We calculate the masses of the members of the pseudoscalar meson octet to next-to-leading order (NLO). The small isospin mass splitting $\Delta m=m_{\mathrm{d}}-m_{\mathrm{u}}$ is included in order $\Delta m$, and lattice effects are included in order $a^{2}$.

The purpose of this paper is twofold. On the one hand, the results include the limit of a vanishing twist angle, which is ordinary untwisted lattice QCD with three nondegenerate Wilson quarks. For this case our calculations reveal the effects of the lattice discretisation on the isospin splitting for pseudoscalar meson masses. In addition to that, even the further limit of vanishing lattice spacing $a$ is of interest, as it includes the effects of mixing in next-to-leading order, which are not completely included in the original work [22].

On the other hand, simulations of twisted mass lattice QCD including strange quarks are in practice done by adding a fourth heavy charm quark and implementing full twist in both the up-down and the strange-charm sector in order to ensure automatic $\mathcal{O}(a)$ improvement in all observables. For the case of the masses of pions, which are made of upquarks and downquarks only, it turns out that full twist in the updown sector alone is not sufficient for $\mathcal{O}(a)$ improvement and that the fully twisted strange-charm sector is necessary. Our calculations make the influence of unaccompanied strange quarks on the pion masses explicit and in this way point to the need of including the charm quarks in the simulations.

\section{Chiral Perturbation Theory}

In twisted mass lattice QCD the twist in the quark sector has to be implemented orthogonally to the mass splitting in order to maintain positivity of the fermion determinant $[6,23]$. In this paper we introduce the mass twist in the $\mathrm{u}$ - $\mathrm{d}$ sector via the third Gell-Mann-matrix $\lambda_{3}$. Consequently the mass splitting is implemented by means of $\lambda_{1}$. The strange quark is introduced as a standard Wilson fermion. The three-flavour quark mass matrix is thus given by

$$
\begin{aligned}
M & =\widehat{m} \mathbf{1}+\left(m_{\mathrm{s}}-\widehat{m}\right)\left(\frac{1}{3} \mathbf{1}-\frac{1}{\sqrt{3}} \lambda_{8}\right)+\frac{1}{2} \Delta m \lambda_{1} \\
& =\left(\begin{array}{ccc}
\widehat{m} & \frac{1}{2} \Delta m & 0 \\
\frac{1}{2} \Delta m & \widehat{m} & 0 \\
0 & 0 & m_{\mathrm{s}}
\end{array}\right), \\
\widehat{m} & \doteq \frac{m_{\mathrm{u}}+m_{\mathrm{d}}}{2}, \\
\Delta m & \doteq m_{\mathrm{d}}-m_{\mathrm{u}} .
\end{aligned}
$$

The chiral rotation entailing the twisted mass is given by

$$
\begin{aligned}
M & \longrightarrow \exp \left[\mathrm{i} \gamma_{5} \lambda_{3} \frac{\omega}{2}\right] M \exp \left[\mathrm{i} \gamma_{5} \lambda_{3} \frac{\omega}{2}\right] \\
& =\left(\begin{array}{ccc}
\widetilde{m}+\mathrm{i} \gamma_{5} \mu & \frac{1}{2} \Delta m & 0 \\
\frac{1}{2} \Delta m & \widetilde{m}-\mathrm{i} \gamma_{5} \mu & 0 \\
0 & 0 & m_{\mathrm{s}}
\end{array}\right), \\
\mu & =\widehat{m} \sin (\omega), \\
\widetilde{m} & =\widehat{m} \cos (\omega), \\
\omega & =\arctan \left(\frac{\mu}{\widetilde{m}}\right) .
\end{aligned}
$$

The effective Lagrangian describing the physics of the pseudoscalar mesons is constructed via the power-counting scheme of [24], accounting for terms of order $\{m, a\}$ in leading order (LO) and $\left\{m^{2}, m a, a^{2}\right\}$ in next-to-leading order (NLO), respectively. The Lagrangian is formulated in terms of the matrix-valued field:

$$
U \doteq \exp \left[\frac{\mathrm{i}}{F_{0}} \sum_{a=1}^{8} \lambda_{a} \phi_{a}\right] .
$$

It contains the meson fields $\phi_{a}$ and the low-energy constant (LEC) $F_{0}$, which equals the pion decay constant for leading order. The quark masses and the lattice spacing $a$ enter the Lagrangian through the variables

$$
\begin{aligned}
& \chi \doteq 2 B_{0} M, \\
& \rho \doteq \rho_{0} \mathbf{1}=2 W_{0} a \mathbf{1},
\end{aligned}
$$

where $B_{0}$ and $W_{0}$ are additional LECs. For leading order the chiral effective Lagrangian reads as follows:

$$
\begin{aligned}
\mathscr{L}_{\mathrm{LO}}= & \frac{F_{0}^{2}}{4} \operatorname{Tr}\left(\partial_{\mu} U \partial_{\mu} U^{\dagger}\right)-\frac{F_{0}^{2}}{4} \operatorname{Tr}\left(\chi U^{\dagger}+U \chi^{\dagger}\right) \\
& -\frac{F_{0}^{2}}{4} \operatorname{Tr}\left(\rho U^{\dagger}+U \rho^{\dagger}\right) .
\end{aligned}
$$

In numerical simulations of twisted mass lattice QCD a chirally rotated mass matrix like (3) has to be used. On the other hand, in chiral perturbation theory it is more convenient to employ the physical basis. It is obtained by applying the inverse of the above chiral rotation, such that the mass matrix retains its original form (1), and the lattice term goes over to

$$
\begin{aligned}
\rho(\omega) & =\exp \left[\mathrm{i} \lambda_{3} \frac{\omega}{2}\right] \rho \exp \left[\mathrm{i} \lambda_{3} \frac{\omega}{2}\right] \\
& =\left(\begin{array}{ccc}
\tilde{\rho}+\mathrm{i} \rho_{3} & 0 & 0 \\
0 & \tilde{\rho}-\mathrm{i} \rho_{3} & 0 \\
0 & 0 & \rho_{0}
\end{array}\right) \\
& =\frac{1}{3}\left(2 \widetilde{\rho}+\rho_{0}\right) \mathbf{1}-\frac{1}{\sqrt{3}}\left(\rho_{0}-\tilde{\rho}\right) \lambda_{8}+\mathrm{i} \rho_{3} \lambda_{3},
\end{aligned}
$$




$$
\begin{aligned}
\widetilde{\rho} & =\rho_{0} \cos (\omega), \\
\rho_{3} & =\rho_{0} \sin (\omega) .
\end{aligned}
$$

In order to set up chiral perturbation theory for the computation of meson masses and other observables, the effective Lagrangian has to be expanded around its minimum. In contrast to the case of continuous space-time, where the minimum is located at vanishing meson fields, $\phi_{a}=0$, its position is shifted for twisted mass lattice QCD and is located at

$$
\begin{aligned}
& \check{\phi}_{i}=0 \text { for } i \neq 3, \\
& \check{\phi}_{3}=\frac{F_{0} \rho_{3}}{\widehat{\chi}+\widetilde{\rho}},
\end{aligned}
$$

and the corresponding value of the field $U$ is

$$
U_{0} \doteq \exp \left[\frac{\mathrm{i}}{F_{0}} \lambda_{3} \check{\phi}_{3}\right] \text {. }
$$

It has turned out that a suitable parameterisation of the field $U$, taking the expansion point $U_{0}$ into account, is given by

$$
U=U_{0}^{1 / 2} U^{\prime} U_{0}^{1 / 2}
$$

such that the minimum of the Lagrangian is now at $U^{\prime}=\mathbf{1}$. Expanding in terms of the shifted meson fields

$$
U^{\prime} \doteq \exp \left[\frac{\mathrm{i}}{F_{0}} \sum_{a=1}^{8} \lambda_{a} \phi_{a}^{\prime}\right]
$$

with the help of the Baker-Campbell-Hausdorff series [25] has the advantage that spurious three-point vertices are eliminated and that just one quartic vertex remains in the Feynman rules, which simplifies the loop calculations a lot.

In order to derive the meson masses in NLO, the effective Lagrangian has to be extended by terms of the next higher chiral dimension. Neglecting external fields and constant terms, this leads to the NLO lattice $\chi$ PT Lagrangian $[10,24]$

$$
\begin{aligned}
\mathscr{L}_{\mathrm{NLO}}= & \mathscr{L}_{\mathrm{LO}}-L_{1}\left[\operatorname{Tr}\left(\partial_{\mu} U \partial_{\mu} U^{\dagger}\right)\right]^{2} \\
& -L_{2}\left[\operatorname{Tr}\left(\partial_{\mu} U \partial_{\nu} U^{\dagger}\right)\right]^{2} \\
& -L_{3} \operatorname{Tr}\left(\left[\partial_{\mu} U \partial_{\mu} U^{\dagger}\right]^{2}\right) \\
& +L_{4} \operatorname{Tr}\left(\partial_{\mu} U \partial_{\mu} U^{\dagger}\right) \operatorname{Tr}\left(\chi^{\dagger} U+U^{\dagger} \chi\right) \\
& +W_{4} \operatorname{Tr}\left(\partial_{\mu} U \partial_{\mu} U^{\dagger}\right) \operatorname{Tr}\left(\rho^{\dagger} U+U^{\dagger} \rho\right) \\
& +L_{5} \operatorname{Tr}\left(\partial_{\mu} U \partial_{\mu} U^{\dagger}\left(U \chi^{\dagger}+\chi U^{\dagger}\right)\right) \\
& +W_{5} \operatorname{Tr}\left(\partial_{\mu} U \partial_{\mu} U^{\dagger}\left(U \rho^{\dagger}+\rho U^{\dagger}\right)\right) \\
& -L_{6}\left[\operatorname{Tr}\left(\chi^{\dagger} U+U^{\dagger} \chi\right)\right]^{2} \\
& -W_{6} \operatorname{Tr}\left(\chi^{\dagger} U+U^{\dagger} \chi\right) \operatorname{Tr}\left(\rho^{\dagger} U+U^{\dagger} \rho\right) \\
& -L_{7}\left[\operatorname{Tr}\left(\chi^{\dagger} U-U^{\dagger} \chi\right)\right]^{2}
\end{aligned}
$$

$$
\begin{aligned}
& -W_{7} \operatorname{Tr}\left(\chi^{\dagger} U-U^{\dagger} \chi\right) \operatorname{Tr}\left(\rho^{\dagger} U-U^{\dagger} \rho\right) \\
& -L_{8} \operatorname{Tr}\left(\chi^{\dagger} U \chi^{\dagger} U+U^{\dagger} \chi U^{\dagger} \chi\right) \\
& -W_{8} \operatorname{Tr}\left(\rho^{\dagger} U \chi^{\dagger} U+U^{\dagger} \rho U^{\dagger} \chi\right) \\
& -W_{6}^{\prime}\left[\operatorname{Tr}\left(\rho^{\dagger} U+U^{\dagger} \rho\right)\right]^{2} \\
& -W_{7}^{\prime}\left[\operatorname{Tr}\left(\rho^{\dagger} U-U^{\dagger} \rho\right)\right]^{2} \\
& -W_{8}^{\prime} \operatorname{Tr}\left(\rho^{\dagger} U \rho^{\dagger} U+U^{\dagger} \rho U^{\dagger} \rho\right)
\end{aligned}
$$

The masses in NLO receive tree-level contributions from $\mathscr{L}_{\text {NLO }}$ and one-loop contributions from $\mathscr{L}_{\text {LO }}$. The loop contributions produce divergences that require renormalisation of the low-energy coefficients $L_{i}, W_{i}$, and $W_{i}^{\prime}$.

The necessary steps for deriving the meson masses are as follows. Mass matrix (1) and twisted lattice term (8) are inserted in Lagrangian (14), and the resulting expression is expanded in the meson fields $\phi_{a}$ up to linear terms in order to find the minimum. The minimum of the Lagrangian at NLO is given by

$$
\begin{aligned}
\check{\phi}_{3}= & F_{0} \rho_{3} \frac{F_{0}^{2}+8 W_{6}\left(2 \hat{\chi}+\chi_{\mathrm{s}}\right)+8 W_{8} \hat{\chi}}{F_{0}^{2} \hat{\chi}+16 L_{6}\left(2 \hat{\chi}+\chi_{\mathrm{s}}\right) \hat{\chi}+16 L_{8}\left(\hat{\chi}^{2}+\Delta \chi^{2}\right)} \\
& +\mathcal{O}\left(a^{2}\right) .
\end{aligned}
$$

As this quantity enters the calculation only quadratically, its terms $\sim a^{2}$ can be neglected. Next the parameterisation of the fields according to (12) is implemented, such that the minimum of the Lagrangian occurs at the origin of the shifted meson fields. Expanding around this minimum up to quadratic terms in the fields gives us the inverse meson propagator at tree level. For LO the inverse propagator reads as follows:

$$
\mathrm{i} G^{-1}\left(p^{2}\right)=p^{2} \mathbf{1}+\mathfrak{M}_{\mathrm{LO}}
$$

The LO mass squared matrix $\mathfrak{M}_{\text {LO }}$ contains nondiagonal terms that generate mixings in $\pi^{0}-\eta$ sector and among the kaon fields. The loop calculations require the LO propagator and the quartic vertices. The vertices are obtained by expanding the NLO Lagrangian up to quartic terms in the shifted meson fields, which leads to complicated expressions containing momentum dependent terms.

At NLO the inverse meson propagator receives additional tree-level contributions and self-energy terms from the loop diagrams:

$$
\mathrm{i} G^{-1}\left(p^{2}\right)=p^{2} \mathbf{1}+\mathfrak{M}_{\mathrm{LO}}-A+p^{2} B
$$

The matrices $A$ and $B$ are rather complicated and contain various off-diagonal mixing terms. The divergent contributions in the self-energies can be separated from the finite ones and absorbed into the NLO renormalised LECs $L_{i}^{\mathrm{r}}$ via a modified minimal subtraction scheme as shown in [22]. 
Field renormalisation can be achieved by writing the inverse propagator as

$$
\begin{aligned}
\mathrm{i} G^{-1}\left(p^{2}\right)= & \left(1+\frac{B}{2}\right) \\
& \cdot\left[p^{2} 1+\mathfrak{M}_{\mathrm{LO}}-A-\frac{1}{2}\left\{B, \mathfrak{M}_{\mathrm{LO}}\right\}\right] \\
& \cdot\left(1+\frac{B}{2}\right)+\mathcal{O}\left(p^{6}\right),
\end{aligned}
$$

such that the expression in square brackets contains the normalised kinetic term. The squared meson masses are then obtained by diagonalising the matrix $\left[\mathfrak{M}_{\mathrm{LO}}-A-\right.$ $\left.(1 / 2)\left\{B, \mathfrak{M}_{\mathrm{LO}}\right\}\right]$.

\section{Results for the Isospin Splittings}

For LO in the limit $\Delta m \rightarrow 0$ the method outlined above reproduces the known results [14]:

$$
\begin{aligned}
\bar{m}_{\pi}^{2} & =2 B_{0} \widehat{m}+2 a W_{0} \cos (\omega)+\frac{a^{2} W_{0}^{2} \sin ^{2}(\omega)}{B_{0} \widehat{m}} \\
& +\mathcal{O}\left(a^{3}\right), \\
\bar{m}_{K}^{2} & =B_{0}\left(\widehat{m}+m_{\mathrm{s}}\right)+a W_{0}(1+\cos (\omega))+\frac{1}{2} \\
& \cdot \frac{a^{2} W_{0}^{2} \sin ^{2}(\omega)}{B_{0} \widehat{m}}+\mathcal{O}\left(a^{3}\right), \\
\bar{m}_{\eta}^{2} & =\frac{1}{3}\left[2 B_{0}\left(\widehat{m}+2 m_{\mathrm{s}}\right)+2 a W_{0}(2+\cos (\omega))\right. \\
& \left.+\frac{a^{2} W_{0}^{2} \sin ^{2}(\omega)}{B_{0} \widehat{m}}\right]+\mathcal{O}\left(a^{3}\right) .
\end{aligned}
$$

We list these expressions here since they are used as convenient abbreviations in the NLO results.

With our choice of the generators for the quark mass splitting and twist, the neutral pion is $\pi_{1} \equiv \pi^{0}$, and the charged pions $\pi^{ \pm}$are linear combinations of the mass eigenstates $\pi_{2}$ and $\pi_{3}$. The charged pion $\pi_{2}$ represents the component that does not mix with $\eta$ and is "orthogonal" to twist $\left(\lambda_{3}\right)$ and to mass splitting $\left(\lambda_{1}\right)$ and is therefore least affected by these factors. The mass of the charged pion $\pi_{2}$ is given in NLO by

$$
\begin{aligned}
M_{\pi_{2}}^{2} & =\bar{m}_{\pi}^{2}+\frac{32 B_{0}^{2}}{F_{0}^{2}}\left[\left(-2 L_{4}^{\mathrm{r}}-L_{5}^{\mathrm{r}}+4 L_{6}^{\mathrm{r}}+2 L_{8}^{\mathrm{r}}\right) \widehat{m}^{2}\right. \\
& \left.+\left(-L_{4}^{\mathrm{r}}+2 L_{6}^{\mathrm{r}}\right) \widehat{m} m_{\mathrm{s}}\right]+\frac{32 a B_{0} W_{0}}{F_{0}^{2}}\left[\left(-W_{4}^{\mathrm{r}}+W_{6}^{\mathrm{r}}\right) \widehat{m}\right. \\
& +\left(-L_{4}^{\mathrm{r}}+W_{6}^{\mathrm{r}}\right) m_{\mathrm{s}} \cos (\omega) \\
& +\left(-2 L_{4}^{\mathrm{r}}-L_{5}^{\mathrm{r}}-2 W_{4}^{\mathrm{r}}-W_{5}^{\mathrm{r}}+4 W_{6}^{\mathrm{r}}+2 W_{8}^{\mathrm{r}}\right) \widehat{m}
\end{aligned}
$$

$$
\begin{aligned}
& \cdot \cos (\omega)]+\frac{32 a^{2} W_{0}^{2}}{F_{0}^{2}}\left[-2 W_{4}^{\mathrm{r}}-W_{5}^{\mathrm{r}}+4 W_{6}^{\prime \mathrm{r}}+2 W_{8}^{\prime \mathrm{r}}\right. \\
& +\left(-W_{4}^{\mathrm{r}}+2 W_{6}^{\prime \mathrm{r}}\right) \cos (\omega) \\
& +\left(-4 L_{6}^{\mathrm{r}}-2 L_{8}^{\mathrm{r}}+4 W_{6}^{\mathrm{r}}+2 W_{8}^{\mathrm{r}}-4 W_{6}^{\prime \mathrm{r}}-2 W_{8}^{\prime \mathrm{r}}\right) \\
& \left.\cdot \sin ^{2}(\omega)+\left(-L_{4}^{\mathrm{r}}-2 L_{6}^{\mathrm{r}}+2 W_{6}^{\mathrm{r}}\right)\left[\frac{m_{\mathrm{s}}}{2 \widehat{m}}\right] \sin ^{2}(\omega)\right] \\
& +\frac{\bar{m}_{\pi}^{2}}{32 \pi^{2} F_{0}^{2}}\left[\bar{m}_{\pi}^{2} \ln \left(\frac{\bar{m}_{\pi}^{2}}{\Lambda^{2}}\right)-\frac{\bar{m}_{\eta}^{2}}{3} \ln \left(\frac{\bar{m}_{\eta}^{2}}{\Lambda^{2}}\right)\right] \\
& +\mathcal{O}\left(\Delta m^{3}, a^{3}\right) .
\end{aligned}
$$

In NLO the pions appear to lose their automatic $\mathcal{O}(a)$ improvement at full twist, due to the presence of the term proportional to $\left(-W_{4}^{\mathrm{r}}+W_{6}^{\mathrm{r}}\right) a$. This term stems from the presence of the strange quark and is independent of $\omega$.

We also note that in the pion masses dependencies on the strange quark mass $m_{\mathrm{s}}$ show up that are independent of $\omega$ and persist in the continuum limit. As the pions are made from up- and downquarks, this term proportional to $m_{\mathrm{s}}$ is counterintuitive. In the context of QCD in the continuum it has its origin in the fermion determinant or, in terms of Feynman diagrams, in strange quark loops that lead to NLO terms in the effective Lagrangian. The associated LECs $L_{4}^{\mathrm{r}}$ and $L_{6}^{\mathrm{r}}$ have very small empirical values, however [26].

In the calculation of the NLO pion masses for nondegenerate quark masses we find that the mass splitting between the neutral pion and the charged pion $\pi_{2}$ is induced by the $\pi^{0}-\eta$ mixing and surprisingly appears to be at least of order $\Delta m^{2}$. These terms are manageable in the tree-level contributions but lead to quite complicated dependences in the loop contributions, in particular in the $\mathcal{O}\left(a^{2}\right)$ terms. Since the quark mass difference $\Delta m^{2}$ is very small, we decide to list the pion mass splittings including the $\Delta m^{2}$ terms up to order $a$ only, neglecting $\mathcal{O}\left(a^{2}\right)$ terms. Then the mass difference between the neutral pion $\pi_{1}$ and the charged pion $\pi_{2}$ is

$$
\begin{aligned}
& M_{\pi_{1}}^{2}-M_{\pi_{2}}^{2}=B_{0} \Delta m^{2}\left\{\frac{1}{4\left(m_{\mathrm{s}}-\widehat{m}\right)}[1\right. \\
& \left.-\frac{a W_{0}(1-\cos \omega)}{B_{0}\left(m_{\mathrm{s}}-\widehat{m}\right)}\right]+\frac{4 B_{0}}{3 F_{0}^{2}}\left(-3 L_{4}^{\mathrm{r}}+2 L_{5}^{\mathrm{r}}+6 L_{6}^{\mathrm{r}}\right. \\
& \left.-78 L_{7}^{\mathrm{r}}-18 L_{8}^{\mathrm{r}}\right)+\frac{4 B_{0}}{F_{0}^{2}}\left(\frac{\widehat{m}}{m_{\mathrm{s}}-\widehat{m}}\right)\left(-3 L_{4}^{\mathrm{r}}-2 L_{5}^{\mathrm{r}}\right. \\
& \left.+6 L_{6}^{\mathrm{r}}\right)+\frac{4 a W_{0}}{F_{0}^{2}\left(m_{\mathrm{s}}-\widehat{m}\right)}\left[\left(-L_{5}^{\mathrm{r}}-3 W_{4}^{\mathrm{r}}-W_{5}^{\mathrm{r}}+3 W_{6}^{\mathrm{r}}\right)\right. \\
& +\left(L_{4}^{\mathrm{r}}+L_{5}^{\mathrm{r}}+20 L_{7}^{\mathrm{r}}+4 L_{8}^{\mathrm{r}}+2 W_{4}^{\mathrm{r}}+W_{5}^{\mathrm{r}}-3 W_{6}^{\mathrm{r}}\right. \\
& \left.-10 W_{7}^{\mathrm{r}}-2 W_{8}^{\mathrm{r}}\right)(1-\cos \omega)+\left(\frac{\widehat{m}}{m_{\mathrm{s}}-\widehat{m}}\right)\left(3 L_{4}^{\mathrm{r}}\right. \\
& \left.\left.+L_{5}^{\mathrm{r}}+4 L_{8}^{\mathrm{r}}+W_{5}^{\mathrm{r}}-3 W_{6}^{\mathrm{r}}-2 W_{8}^{\mathrm{r}}\right)(1-\cos \omega)\right]
\end{aligned}
$$




$$
\begin{aligned}
& -\frac{B_{0}}{64 \pi^{2} F_{0}^{2}}\left[\ln \left(\frac{\bar{m}_{K}^{2}}{\Lambda^{2}}\right)+1\right] \\
& -\frac{1}{192 \pi^{2} F_{0}^{2}\left(m_{\mathrm{s}}-\widehat{m}\right)}\left[1-\frac{a W_{0}(1-\cos \omega)}{B_{0}\left(m_{\mathrm{s}}-\widehat{m}\right)}\right]\left[\bar{m}_{\pi}^{2}\right. \\
& \cdot \ln \left(\frac{\bar{m}_{\pi}^{2}}{\Lambda^{2}}\right)-3 \bar{m}_{\eta}^{2} \ln \left(\frac{\bar{m}_{\eta}^{2}}{\Lambda^{2}}\right)-\frac{1}{2}\left(3 \bar{m}_{\eta}^{2}-\bar{m}_{\pi}^{2}\right) \\
& \left.\left.\cdot \ln \left(\frac{\bar{m}_{K}^{2}}{\Lambda^{2}}\right)+\frac{3}{2}\left(\bar{m}_{\pi}^{2}+\bar{m}_{\eta}^{2}\right)\right]\right\}+\mathcal{O}\left(\Delta m^{3}, a^{2}\right) .
\end{aligned}
$$

We observe that at full twist, $\cos \omega=0$, also terms of order $a$ are present. They are not proportional to $m_{\mathrm{s}}$. The presence of these terms is a consequence of the fact that the strange quark is not a member of a twisted doublet. Namely, if the strange quark would be accompanied by a fourth quark, forming together a fully twisted doublet, automatic $\mathcal{O}(a)$ improvement would forbid the existence of such terms.

Due to the mass twist the charged pions represented by the fields $\pi_{2}$ and $\pi_{3}$ also gain different masses, which leads to the splitting

$$
\begin{aligned}
& M_{\pi_{3}}^{2}-M_{\pi_{2}}^{2}=\frac{32 a^{2} W_{0}^{2}}{F_{0}^{2}}\left(-4 L_{6}^{\mathrm{r}}-2 L_{8}^{\mathrm{r}}+4 W_{6}^{\mathrm{r}}+2 W_{8}^{\mathrm{r}}\right. \\
& \left.-4 W_{6}^{\prime \mathrm{r}}-2 W_{8}^{\prime \mathrm{r}}\right) \sin ^{2}(\omega)+\mathcal{O}\left(a^{3}\right) .
\end{aligned}
$$

This mass difference is a pure lattice artifact that vanishes in the continuum limit $a \rightarrow 0$.

We have also calculated the mass splitting between the kaon masses in NLO including terms of order $a^{2}$. As the characteristic features show up at order $a$ already, we display the mass splitting between neutral kaons $K_{6}$ and $K_{7}$ and charged kaons $K_{4}$ and $K_{5}$, including terms linear in $a$, which is

$$
\begin{aligned}
M_{K^{0}}^{2}-M_{K^{ \pm}}^{2}=B_{0} \Delta m\{1 \\
+\frac{16 B_{0}}{F_{0}^{2}}\left[\left(-2 L_{4}^{\mathrm{r}}-L_{5}^{\mathrm{r}}+4 L_{6}^{\mathrm{r}}+2 L_{8}^{\mathrm{r}}\right) \widehat{m}\right. \\
\left.+\left(-L_{4}^{\mathrm{r}}-L_{5}^{\mathrm{r}}+2 L_{6}^{\mathrm{r}}+2 L_{8}^{\mathrm{r}}\right) m_{\mathrm{s}}\right] \\
+\frac{8 a W_{0}}{F_{0}^{2}}\left[\left(-2 L_{5}^{\mathrm{r}}-6 W_{4}^{\mathrm{r}}-2 W_{5}^{\mathrm{r}}+6 W_{6}^{\mathrm{r}}+4 W_{8}^{\mathrm{r}}\right)\right. \\
\left.+\left(L_{5}^{\mathrm{r}}+4 W_{4}^{\mathrm{r}}+W_{5}^{\mathrm{r}}-4 W_{6}^{\mathrm{r}}-2 W_{8}^{\mathrm{r}}\right)(1-\cos \omega)\right] \\
+\frac{1}{32 \pi^{2} F_{0}^{2}}\left[\frac{2 \bar{m}_{\eta}^{2}}{3} \ln \left(\frac{\bar{m}_{\eta}^{2}}{\Lambda^{2}}\right)+\frac{1}{B_{0}\left(m_{\mathrm{s}}-\widehat{m}\right)}\right. \\
\quad \frac{\bar{m}_{K}^{2}}{2}\left[\bar{m}_{\eta}^{2} \ln \left(\frac{\bar{m}_{\eta}^{2}}{\Lambda^{2}}\right)-\bar{m}_{\pi}^{2} \ln \left(\frac{\bar{m}_{\pi}^{2}}{\Lambda^{2}}\right)\right] \\
\left.\left.+\left(1-\frac{a W_{0}(1-\cos \omega)}{B_{0}\left(m_{\mathrm{s}}-\widehat{m}\right)}\right)\right]\right\}+\mathcal{O}\left(\Delta m^{2}, a^{2}\right) .
\end{aligned}
$$

It vanishes in the limit $\Delta m=0$, as it should. In the continuum limit the expression reduces to that given in [22].

In contrast to the case of pions, the kaon mass splitting has contributions linear in $\Delta m$, part of which is produced by pion and eta loops. It is thus significantly more sensitive to the tiny quark mass difference $\Delta m$ than the pion mass splitting. Therefore the kaon masses represent observables ideally suited for numerical investigations of the quark mass difference $\Delta m$ in lattice QCD. For studies with three flavours of the Wilson quarks the formula shows the lattice artifacts proportional to $a \Delta m$.

We have also obtained the expression for the mass of the $\eta$ meson in the case of nondegenerate quark masses. It is quite lengthy, and as it does not reveal any new characteristic features compared to the pion masses, we refrain from presenting it here.

\section{Conclusions}

To summarise, the masses of the pseudoscalar mesons for the case of nondegenerate upquarks, downquarks, and strange quarks have been obtained in chiral perturbation theory for twisted mass lattice QCD including lattice terms quadratic in the lattice spacing $a$ and in $\Delta m$. Automatic $\mathcal{O}(a)$ improvement of the pion masses at maximal twist in the up-down sector is lost. At maximal twist, terms of order $a$ are present, which have two different origins. On the one hand, at NLO the pion masses get an equal contribution of order $a$ that stems from the presence of the strange quark in the effective Lagrangian. On the other hand, the inverse propagator contains offdiagonal terms that represent a mixing between the neutral pion and the $\eta$ meson. These yield a contribution to the neutral pion mass which is proportional to $\Delta m^{2}$. Apart from continuum terms $\left(a^{0}\right)$ it has terms of order $a$ that spoil automatic $\mathcal{O}(a)$ improvement at maximal twist. We present the mass splitting between neutral and charged pions including terms of orders $\Delta m^{2}$ and $a$. In addition, the twisted mass generates a mass difference between the charged pions of order $a^{2}$.

Finally, the lattice corrections to the mass splitting between neutral and charged kaons, which are proportional to $\Delta m$, are presented including terms of order $a$. In the kaon sector there is also mixing, in this case between $K_{4}$ and $K_{6}$ and between $K_{5}$ and $K_{7}$. As for the pions, it leads to terms of order $a$ that spoil automatic $\mathcal{O}(a)$ improvement.

If the strange quark were introduced with a partner (charm quark), associated with another twist in this heavy sector, the lattice artifacts of order $a$ and higher would look quite different. In particular, full twist in both sectors would ensure absence of all $\mathcal{O}(a)$ terms. Our results make the idea that such inclusion of a twisted fourth quark is advantageous for reducing unwanted lattice artifacts explicit.

\section{Competing Interests}

The authors declare that there are no competing interests regarding the publication of this paper. 


\section{References}

[1] S. R. Sharpe, "Applications of chiral perturbation theory to lattice QCD," http://arxiv.org/abs/hep-lat/0607016.

[2] M. Golterman, "Applications of chiral perturbation theory to lattice QCD," in Modern Perspectives in Lattice QCD: Quantum Field Theory and High Performance Computing, L. Lellouch, R. Sommer, B. Svetitsky, A. Vladikas, and L. F. Cugliandolo, Eds., vol. 93 of Lecture Notes of the Les Houches Summer School, Oxford University Press, 2011.

[3] R. Frezzotti, P. A. Grassi, S. Sint, and P. Weisz, "A local formulation of lattice QCD without unphysical fermion zero modes," Nuclear Physics B-Proceedings Supplements, vol. 83-84, no. 1-3, pp. 941-946, 2000.

[4] R. Frezzotti, P. A. Grassi, S. Sint, and P. Weisz, "Lattice QCD with a chirally twisted mass term," Journal of High Energy Physics, vol. 2001, no. 8, p. 58, 2001.

[5] R. Frezzotti and G. C. Rossi, "Chirally improving Wilson fermions 1. $O(a)$ improvement," Journal of High Energy Physics, vol. 2004, article 007, 2004.

[6] R. Frezzotti and G. C. Rossi, "Twisted-mass lattice QCD with mass non-degenerate quarks," Nuclear Physics B-Proceedings Supplements, vol. 128, pp. 193-202, 2004.

[7] A. Shindler, “Twisted mass lattice QCD," Physics Reports, vol. 461, no. 2-3, pp. 37-110, 2008.

[8] R. Baron, Ph. Boucaud, J. Carbonell et al., "Light hadrons from lattice QCD with light $(u, d)$, strange and charm dynamical quarks," Journal of High Energy Physics, vol. 2010, article 111, 2010.

[9] K. Ottnad, C. Michael, S. Reker et al., " $\eta$ and $\eta$ ' mesons from $N_{f}=2+1+1$ twisted mass lattice QCD," Journal of High Energy Physics, vol. 2012, no. 11, article 48, 2012.

[10] G. Münster and C. Schmidt, "Chiral perturbation theory for lattice QCD with a twisted mass term," Europhysics Letters, vol. 66, no. 5, pp. 652-656, 2004.

[11] G. Münster, "On the phase structure of twisted mass lattice QCD," Journal of High Energy Physics, vol. 2004, no. 9, article 035, 2004.

[12] L. Scorzato, "Pion mass splitting and phase structure in twisted mass QCD," The European Physical Journal C: Particles and Fields, vol. 37, no. 4, pp. 445-455, 2004.

[13] S. R. Sharpe and J. M. S. Wu, "Twisted mass chiral perturbation theory at next-to-leading order," Physical Review D, vol. 71, no. 7, Article ID 074501, 19 pages, 2005.

[14] G. Münster and T. Sudmann, "Twisted mass lattice QCD with non-degenerate quark masses," Journal of High Energy Physics, vol. 2006, no. 8, article 085, 2006.

[15] G. Münster and T. Sudmann, "Twisted mass chiral perturbation theory for 2+1+1 quark flavours," Journal of High Energy Physics, vol. 2011, article 116, 2011.

[16] O. Bär and B. Hörz, "Charmless chiral perturbation theory for $N_{f}=2+1+1$ twisted mass lattice QCD," Physical Review D, vol. 90, no. 3, Article ID 034508, 2014.

[17] T. Blum, R. Zhou, T. Doi et al., "Electromagnetic mass splittings of the low lying hadrons and quark masses from $2+1$ flavour lattice QCD + QED," Physical Review D, vol. 82, Article ID 094508, 2010

[18] S. Aoki, K.-I. Ishikawa, N. Ishizuka et al., "1+1+1 flavour QCD + QED simulation at the physical point," Physical Review D, vol. 86, no. 3, Article ID 034507, 2012.
[19] S. Borsanyi, S. Durr, Z. Fodor et al., "Ab initio calculation of the neutron-proton mass difference," Science, vol. 347, no. 6229, pp. 1452-1455, 2015.

[20] T. Ishikawa, T. Blum, M. Hayakawa, T. Izubuchi, C. Jung, and R. Zhou, "Full QED+QCD low-energy constants through reweighting," Physical Review Letters, vol. 109, no. 7, Article ID 072002, 2012.

[21] R. Horsley, Y. Nakamura, D. Pleiter et al., "Electromagnetic splitting of quark and pseudoscalar meson masses from dynamical QCD + QED," in Proceedings of the 31st International Symposium on Lattice Field Theory (Lattice '13), vol. 499, 2014.

[22] J. Gasser and H. Leutwyler, "Chiral perturbation theory: expansions in the mass of the strange quark," Nuclear Physics, Section $B$, vol. 250, no. 1-4, pp. 465-516, 1985.

[23] R. Frezzotti, "Twisted mass lattice QCD," Nuclear Physics B: Proceedings Supplements, vol. 140, pp. 134-140, 2005.

[24] O. Bär, G. Rupak, and N. Shoresh, "Chiral perturbation theory at $\mathcal{O}\left(a^{2}\right)$ for lattice QCD," Physical Review D, vol. 70, no. 3, Article ID 034508, 12 pages, 2004.

[25] M. W. Reinsch, "A simple expression for the terms in the BakerCampbell-Hausdorff series," Journal of Mathematical Physics, vol. 41, no. 4, pp. 2434-2442, 2000.

[26] J. Bijnens and G. Ecker, "Mesonic low-energy constants," Annual Review of Nuclear and Particle Science, vol. 64, no. 1, pp. 149-174, 2014. 

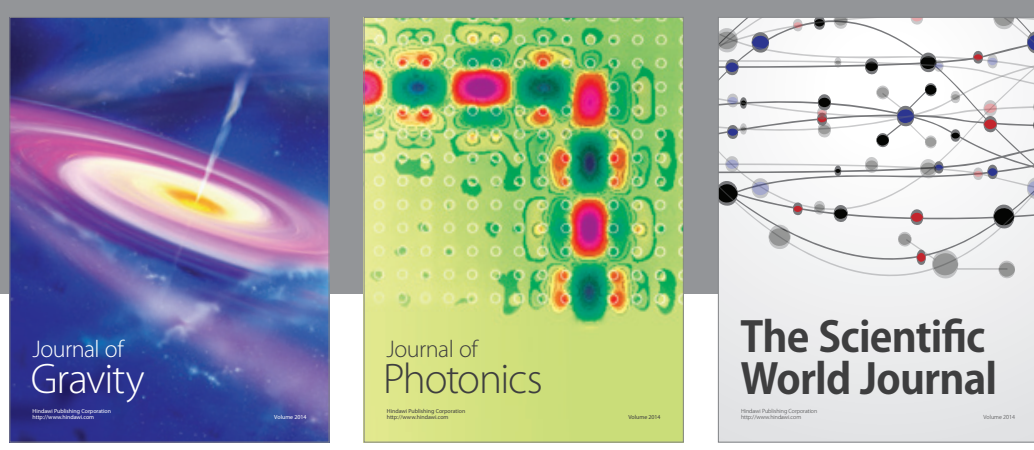

The Scientific World Journal
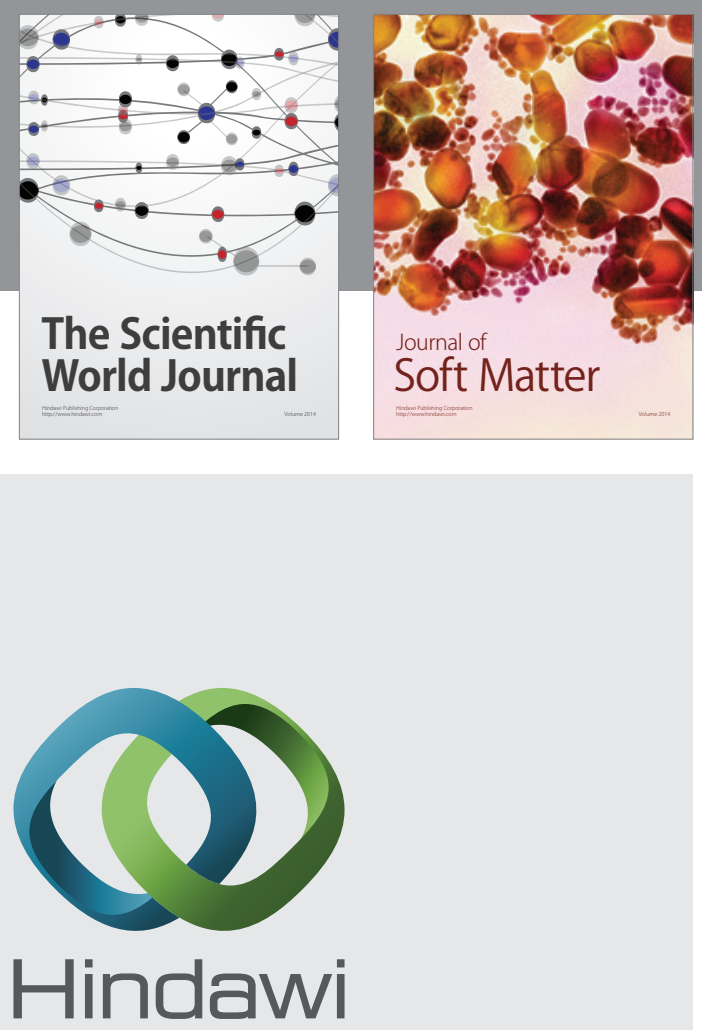

Submit your manuscripts at

http://www.hindawi.com

nternational Journal of

Statistical Mechanics
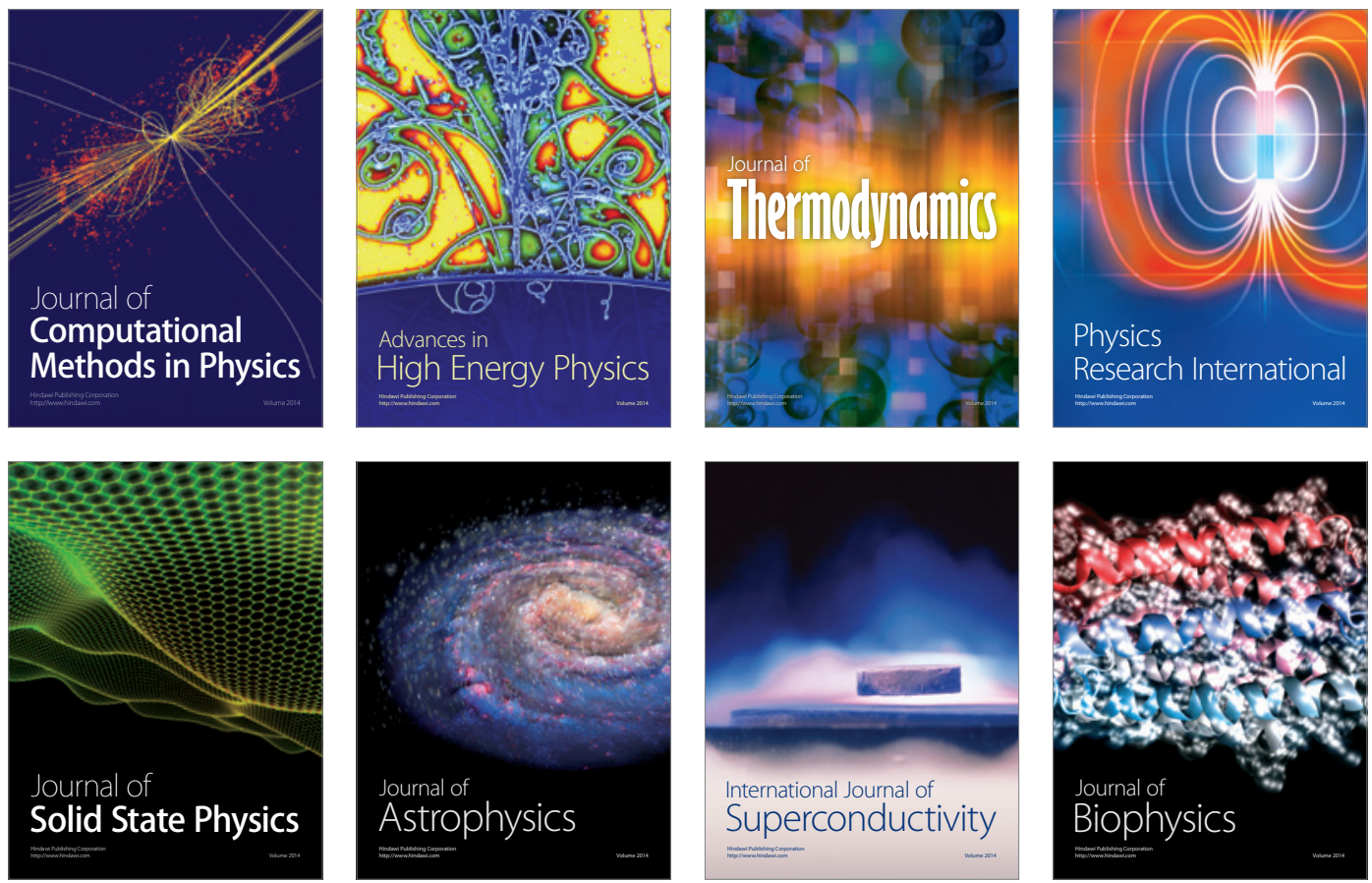
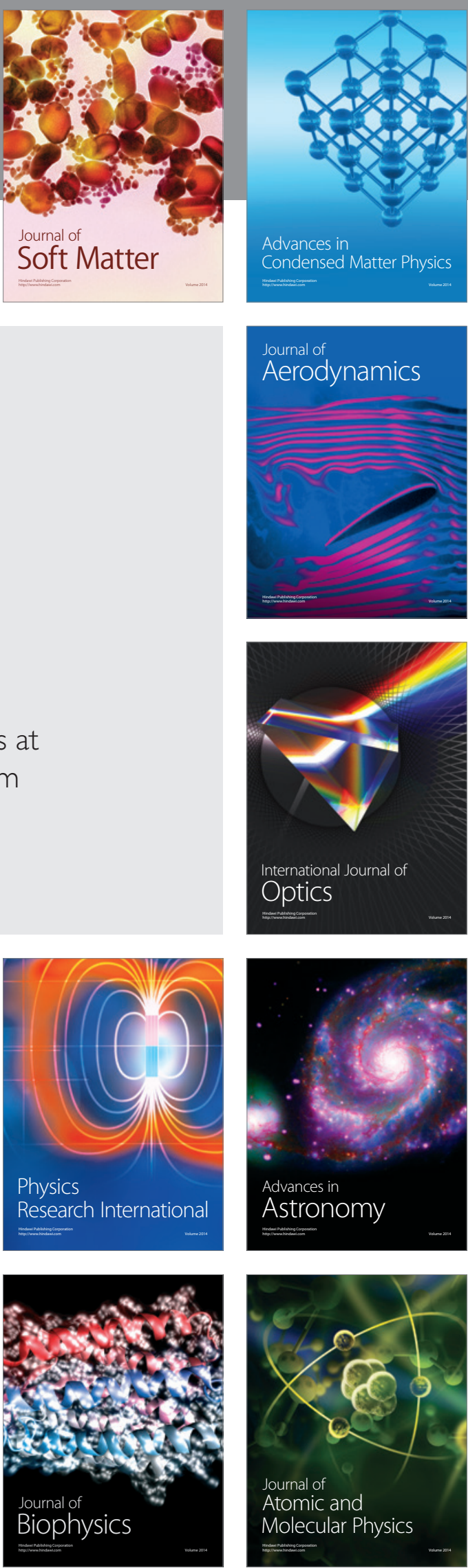